\title{
Simultaneous coronary sinus and left bundle area pacing conferred cardiac resynchronization super response after either alone failed
}

\author{
Xiaoke $\mathrm{Liu}^{1}$ and Yong-Mei Cha ${ }^{2}$ \\ ${ }^{1}$ Borgess Heart Insititue \\ ${ }^{2}$ Mayo Clinic
}

October 1, 2020

\begin{abstract}
Background: A patient with ischemic cardiomyopathy and left bundle branch block (LBBB) who did not respond to standard bi-ventricular pacing underwent attempted left bundle area (LBA) pacing to achieve better cardiac resynchronization (CRT). Methods: LBA pacing failed to correct the LBBB or narrow the QRS. However, simultaneous pacing through the coronary sinus (CS) and LBA pacing leads resulted in marked shortening of the QRS Results: The patient's EF improved from $30 \%$ to $45 \%$ two months post procedure and heart failure symptoms improved by one functional class. Conclusions: Simultaneous LBA and CS pacing may improve clinical outcomes compared to either alone.
\end{abstract}

\section{Introduction:}

Despite advances in the implant technique over the last a few decades, optimal cardiac synchronization and positive response can still not be achieved in about $30 \%$ of the patients. ${ }^{1}$ More recently, conduction system pacing especially left bundle area (LBA) pacing has rapidly emerged as a potential new alternative to traditional bi-ventricular pacing through the coronary sinus (CS) and has been used in certain cases to improve the CRT response in patients who failed conventional CRT (figure 1). ${ }^{2-4}$ Nevertheless, even with LBA pacing, there are still significant number of cases where underlying LBBB cannot be corrected. ${ }^{2-4}$

We here report a case where a conventional CRT non-responder underwent an apparently "failed" LBA pacing implant as evidenced by the inability to correct the underlying LBBB or significantly shorten the QRS duration. However, simultaneous pacing from the previously implanted coronary sinus lead and the apparently "failed" left bundle pacing lead substantially narrowed the QRS and eventually led to CRT super response.

\section{Clinical case:}

A 65 year-old male patient with a prior history of ischemic cardiomyopathy, EF 30\%, NYHA class III, long-standing persistent atrial fibrillation, and LBBB (QRS duration 176ms). He underwent initial standard bi-ventricular ICD implantation in 2014. He was found to have only one suitable target coronary sinus branch in the posterior to posterolateral position for left ventricular pacing. However, despite programming changes to allow fusion of intrinsic conduction with the CS lead and implantation of the right ventricular (RV) defibrillation lead into a septal position, the post-op QRS duration continues to be relatively wide (168ms). No significant improvement was observed in the LV systolic function or patient's clinical heart failure status post implant. Therefore, the patient was brought back to the Ascension Borgess Hospital EP lab for implantation of a LBA pacing lead.

The patient's left precordium was prepared and draped in usual fashion. The axillary vein was re-accessed through which a deflectable EP catheter was inserted and used to map the distal his bundle position. After 
successful registration of the distal his bundle location, a Medtronic his-bundle delivery sheath was advanced and placed in the vicinity of the left bundle area defined as an area about 1-1.5 cm distal from the recorded distal his bundle signal, towards the left ventricular apex (figure 2). A Medtronic 3830 his bundle pacing lead was advanced and used to engage the LBA. After multiple attempts to screw the lead deep into the septum, we were unable to correct the underlying LBBB (figure 2). However, simultaneous pacing using the CS (pole 3 to pole 4) and the new lead in the LBA resulted in a narrower paced QRS morphology (figure 2). The new pacing configuration resulted in marked narrowing of the paced QRS to $130 \mathrm{~ms}$ as well as significantly decreased QRS amplitude on the ECG (figure 3). The newly implanted LBA pacing lead was connected to the atrial port with pacing mode programmed to DDDR using a minimal AV delay at 25ms through the programmer because it is not compatible with the DF-4 right ventricular pacing port (figure 3).

After the successful CRT system revision, the patient's repeat ECHO two months post procedure showed an increase in EF from $30 \%$ to $45 \%$. Patient's heart failure symptoms improved by one functional class.

\section{Discussion}

Conduction system pacing especially LBA pacing has recently gained substantial traction as an alternative to traditional coronary sinus pacing for CRT. ${ }^{2-4}$ There are several potential advantages of LBA pacing that have been demonstrated in multiple studies. Numerous reports have shown impressive QRS shortening and improvement in heart failure together with good pacing parameters in patients who previously failed standard CS pacing and continued to have wide QRS post standard biventricular pacing. ${ }^{2-4}$ However, there are still significant number of patients in whom LBA cannot correct the underlying LBBB, like the case presented here.

The most interesting and somewhat unexpected finding of this case is that when combined with the coronary sinus pacing lead located in the posterolateral position, the apparently "failed" LBA pacing lead resulted in marked shortening of the paced QRS duration, improved electric synchronization and led to CRT superresponse. A recent study has demonstrated that incorporation of an CS lead into an existing functional LBA pacing system may result in additional QRS shortening compared to LBA pacing alone. ${ }^{5}$ However, our case appears to be the first one that showed even a "failed" LBA pacing lead, when combined with an existing CS lead that failed to confer CRT benefit, may result in marked synergistic shortening of the QRS and ultimately improved clinical outcome.

The exact mechanism underlying this synergistic QRS shortening between the CS lead and LBA pacing lead is not entirely clear. Because the LBA pacing lead in this case failed to correct the LBBB and significantly narrow the QRS, the added benefit of pacing through this lead versus the more apically located RV defibrillation lead may be related to the anatomic position of the LBA lead in relation to the CS lead. Having the RV lead in a basal and anterior position creates excellent separation from the CS lead located in the posterolateral aspect of the left ventricle on fluoroscopic views (figure 2). The pacing vector between the CS and LBA leads appears to cover a much larger bulk of left ventricle compared to the one between the CS and RV defibrillation lead.

In conclusion, simultaneous LBA and CS pacing (in the posterolateral position) may achieve substantial synergy and improve CRT outcome in patients who failed either LBA and/or CS pacing alone. We are currently in the process of conducting a larger cohort study to help further confirm this finding and elucidate the underlying mechanism.

\section{Acknowledgement:}

We thank Kyle Liu from Portage Central High School, Kalamazoo, Michigan for his excellence assistance in the graphics that helped improve the paper

\section{References:}

1. Linde C, Ellenbogen K, McAlister FA. Cardiac resynchronization therapy (CRT): clinical trials, guidelines, and target populations.Heart Rhythm 2012;9:S3-S13. 
2. Jiang Z, Chang Q, Wu Y, Ji L,Zhou X, Shan Q. Typical BBB morphology and implantation depth of 3830 electrode predict QRS correction by left bundle branch area pacing. Pacing Clin Electrophysiol 2020;43:110-117

3. Huang W, Su L, Wu S, et al. A novel pacing strategy with low and stable output: Pacing the left bundle branch immediately beyond the conduction block. Can J Cardiol 2017;33:e1731-1736

4. Vijayaraman P, Subzposh FA, Naperkowski A, et al. Prospective evaluation of feasibility, electrophysiologic and echocardiographic characteristics of left bundle branch area pacing. Heart Rhythm 2019;16:1774-1782

5. Zhang W, Huang J, Qi Y, Wang F, et al. Cardiac resynchronization therapy by left bundle branch area pacing in heart failure patients with left bundle branch block. Heart Rhythm 2019;16:1783- 1790.

\section{Figure Legends:}

Figure 1. An example of successful left bundle area (LBA) pacing lead implant performed at Ascension Borgess Hospital in a patient who failed conventional coronary sinus lead implant.

Figure 2. Fluoroscope views of LBA lead and coronary sinus (CS) lead positions. 2A: right anterior oblique view. 2B: left anterior oblique view.

Figure 3. Marked QRS shortening and decreased ST deviation by simultaneous pacing of the LBA and CS leads (pole 3 to pole 4). 3A: standard biventricular pacing produced minimal shorting of the QRS. 3C: LBA pacing failed to correct the baseline LBBBB or shorten the QRS. 3D: Simultaneous LBA and CS pacing led to improved electrical resynchronization and QRS shortening of $46 \mathrm{~ms}$ (from $176 \mathrm{~ms}$ to $130 \mathrm{~ms}$ ).

\section{Hosted file}

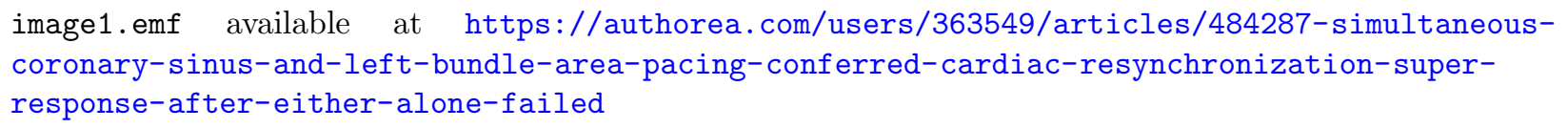

Figure 1

\section{Hosted file}

image2.emf available at https://authorea.com/users/363549/articles/484287-simultaneouscoronary-sinus-and-left-bundle-area-pacing-conferred-cardiac-resynchronization-superresponse-after-either-alone-failed

Figure 2

\section{Hosted file}

image3.emf available at https://authorea.com/users/363549/articles/484287-simultaneouscoronary-sinus-and-left-bundle-area-pacing-conferred-cardiac-resynchronization-superresponse-after-either-alone-failed

Figure 3 